\title{
THE UNITED STATES AND COLOMBIA: UNTYING THE GORDIAN KNOT
}

David Passage

March 2000 